\title{
A Time-Dependent Model of Polar Cap Arcs
}

\author{
L. ZHU, J. J. SOJKA, R. W. SCHUNK, AND D. J. CRAIN
}

Center for Atmospheric and Space Sciences, Utah State University, Logan

\begin{abstract}
A two-dimensional time-dependent model of polar cap arcs has been developed. The electrodynamics of the polar cap arcs are treated self-consistently in the frame of the coupled magnetosphere-ionosphere system. The focus of this paper is to introduce the physics and mathematical formulation of the model and describe the features of the spatial structure and temporal evolution of the polar cap arcs. The modeling results indicate that the time constant for the formation of the polar cap arcs is around $10 \mathrm{~min}$. It is found that an initial single-arc precipitation pattern associated with a polar cap arc tends to split into multiple precipitation regions and leads to a multiple structure of the polar cap arcs. It is also found that strong downward field-aligned currents can develop near the intensive upward field-aligned currents and form a pair structure of the field-aligned current in the polar cap arcs. The model predicts the existence of plasma flow crossing the polar cap arcs, but the amplitude of such a flow is small, and the characteristic time scale of it is much larger than the time constant for the formation of the polar cap arcs. Our results also show that while polar cap arcs are developing, the associated current system tends to become further localized and at steady state the current system in the narrow regions around the arcs is locally self-closed.
\end{abstract}

\section{INTRODUCTION}

The occurrence of auroral arcs at very high geomagnetic latitudes $\left(>80^{\circ}\right)$ had been reported [Weill, 1958; Davis, 1960, 1963; Denholm and Bond, 1961] when the first systematic morphological study of auroras in the oval region began [cf. Akasofu, 1964]. In contrast with the arcs in the auroral oval region which have a good correlation to a southward interplanetary magnetic field (IMF), the auroral arcs at very high latitudes were mostly observed during the periods of northward IMF and quiet magnetic conditions [Berkey et al., 1976; Ismail et al., 1977; Lassen and Danielsen, 1978]. The observed features of the auroral arcs at high-latitude regions have a great diversity. The arcs can be very bright and luminous as they cross the polar cap from the dayside to the nightside of the auroral oval to form a pattern of Greek letter "theta" [Frank et al., 1982, 1986; Nielsen et al., 1990], or they can be confined in the polar cap and remain relatively weak, having multiple discrete patterns and being located either in the evening sector or morning sector of the polar cap [Weber and Buchau, 1981; Ismail and Meng, 1982; Murphree et al., 1982; Gussenhoven, 1982; Carlson et al., 1984; Rich et al., 1990]. Some observations suggest that the arcs at high latitudes are in the regions of open field lines because of the finding of polar rain electrons adjacent to and outside of the arcs [Hardy et al., 1982], but in other observations, the precipitating electrons have the characteristics of the plasma sheet or plasma sheet boundary layers, suggesting that the arcs are on closed field lines [Peterson and Shelley, 1984; Obara et al., 1988]. The convection patterns associated with the arcs can be a mesoscale shear flow pattern [Hoffman et al., 1985; Carlson et al., 1988; Valladares and Carlson, 1991], a largescale sunward flow pattern [Frank et al., 1986], or an ambiguous and confused one [Rich et al., 1990]. One general consensus in the observations of the arcs at high latitudes is that the arcs are associated vith upward field-aligned currents which are carried by energetic precipitating electrons, and the

Copyright 1993 by the American Geophysical Union.

Paper number 92JA01600.

0148-0227/93/92JA-01600\$05.00 energy range of the precipitating electrons spans from a few hundred $\mathrm{eV}$ to a few $\mathrm{keV}$ [Hardy et al., 1982; Valladares and Carlson, 1991]. The dependence of features of the arcs at high latitudes on the $\mathrm{Y}$ component of the IMF has also been reported by many researchers [e.g., Meng, 1981; Gussenhoven, 1982; Makita et al., 1991]. It should be noted that corresponding to the diversity of the observational results, a variety of names have been used for the auroral arcs seen at very high latitudes, such as polar cap arcs, extremely high latitude auroras, Sunaligned arcs, transpolar arcs, and theta auroras. Whether they are the same auroral phenomena at high latitudes and the diversity of the observational results and names is just due to the different nature of the observations, whether they are different auroral manifestations in the ionosphere which have the same magnetospheric driven mechanism, and/or whether they are, indeed, different auroral phenomena which have totally different driven mechanisms are still unclear issues. In the following, we will use the term "polar cap arcs" to refer to the general types of auroral arcs seen at very high latitudes.

Theoretical studies of polar cap arcs are still in very early stages. Most of the relevant theoretical works concern the global-scale ionospheric convection patterns and large-scale ionospheric current configuration during northward IMF and their coupling to the magnetosphere [Russell, 1972; Maezawa, 1976; Burke et al., 1979; Potemra et al., 1984; Chiu et al., 1985; Reiff and Burch, 1985; Burch et al., 1985; Rasmussen and Schunk, 1987; Marklund and Blomberg, 1991]. Only a small portion of the theoretical effort has specifically focused on the structures of the polar cap arcs and their corresponding dynamical processes. Based on S3-2 data, Burke et al. [1982] proposed a model for polar cap arcs occurring on open field lines. By introducing the concept of multiple bifurcation of magnetic field lines, Kan and Burke [1985] constructed a model of polar cap arcs and proposed that theta aurora can occur on the closed field lines, and the less intense polar cap arcs can occur either on closed or open field lines. More recently, Chiu [1989] formulated a model in which polar cap arcs are assumed to be on open field lines and the formation of polar cap arcs is shown to be due to current response to mesoscale velocity shear structures in the ionosphere. It should be noted here that the above theoretical models concerning the structure and the 
corresponding dynamical processes of polar cap arcs are either qualitative, semiquantitative, or steady state models. To date, no time-dependent theoretical model displaying the features of temporal evolution and fine spatial structures of polar cap arcs has been proposed. At the present stage, such a time-dependent model is essential for establishing a more complete integrated temporal and spatial picture of polar cap arcs, thereby providing a guide for synthesizing the diversified observational results and improving our physical understanding of polar cap arcs.

As a first theoretical endeavor in this direction, we have developed a quantitative time-dependent model in which the electrodynamics of polar cap arcs are treated self-consistently in the frame of the coupled magnetosphere-ionosphere system. The goal of developing such a quantitative model is to understand the detailed physics of polar cap arcs and provide a theoretical guide for the diversified observations rather than to fit the modeling results into a specific observational picture. Based on this point, the inputs of the model have been given a maximum flexibility which allows us to simulate the polar cap arcs occurring under various physical conditions. Because of the time-dependent and mesoscale nature of the model, the modeling results can shed light on a series of currently interesting questions of polar cap arcs, such as the following: (1) How do the field-aligned currents of polar cap arcs connect the ionospheric horizontal currents? (2) Is this current system a locally self-closed current system? (3) What is the time constant for the formation of polar cap arcs? (4) How does ionospheric plasma convect in the arc vicinity?

The specific purpose of this paper is to introduce the physics and mathematical formulation of the model and describe the features of spatial structure and temporal evolution of polar cap arcs resulting from a case run.

\section{THEORETICAL MODEL}

Polar cap arcs are neither isolated ionospheric physical processes nor passive ionospheric manifestations of the dynamics occurring in the magnetosphere. The dynamical features of polar cap arcs are coherently determined by the dynamics in the magnetosphere and the ionospheric conditions, in which the ionosphere is not just a passive medium or a "load." This suggests that a self-consistent study of polar cap arcs can be undertaken only in the frame of a coupled magnetosphere-ionosphere (M-I) system. But on the other hand, the coupled M-I system is very complicated and involves a number of dynamical processes. At this stage, a comprehensive model which includes all dynamical processes in the M-I system and can also show the detailed features of polar cap arcs is almost impossible. One important element connecting the dynamics in the magnetosphere and ionosphere is the Alfvén waves propagating along magnetic field lines. A series of work on the M-I coupling via Alfvén waves has been done [Maltsev et al., 1977; Mallinckrodt and Carlson, 1978; Lysak and Dum, 1983; Rothwell et al., 1984; Lysak, 1986, 1991; Kan et al., 1988; Zhu and Kan, 1990], and all of these works concern the auroral dynamics in the oval regions. In this work, we have specifically extended the idea of M-I coupling proposed by Kan et al. [1988] and applied it to the dynamics of polar cap arcs. As a first step we formulate a quantitative model of polar cap arcs which is kept as simple as possible but contains key processes which are treated selfconsistently in the frame of the coupled M-I system. The scenario of processes in the model is described as follows:

Figure 1 is a schematic diagram showing the M-I framework for our polar cap arc model. Initially, a magnetospheric shear flow carried by Alfvén waves propagates toward the ionosphere. The downward propagating Alfvén waves are partially reflected from the ionosphere and then bounce around between the ionosphere and magnetosphere. The features of wave reflections depend on the conditions in the ionosphere and magnetosphere. The propagating Alfvén waves carry both upward and downward field-aligned currents. The precipitating electrons associated with upward field-aligned currents enhance the conductivity in the ionosphere. The changed ionospheric conductivity launches a secondary Alfvén wave toward the magnetosphere. The whole process is transient during which all physical quantities in the ionosphere change selfconsistently in time and polar cap arcs develop. Because of the finite conductivity in the ionosphere, the temporal changes of Alfvén waves in the coupled M-I system diminish with time, and the whole M-I system, as well as the development of polar cap arcs, eventually approaches an asymptotic steady state. At this steady state, the electric field in the ionosphere remains constant in time because there is no further change of the wave field along magnetic field lines, and a constant Poynting flux carried by Alfvén waves feeds the constant energy dissipation in the ionosphere.

A few points in the above brief scenario of the model need further elaboration and justification here. It has been commonly accepted that the major driving force of polar cap arcs is the dynamics in the magnetosphere which influences the ionospheric dynamics mainly via the convection electric field

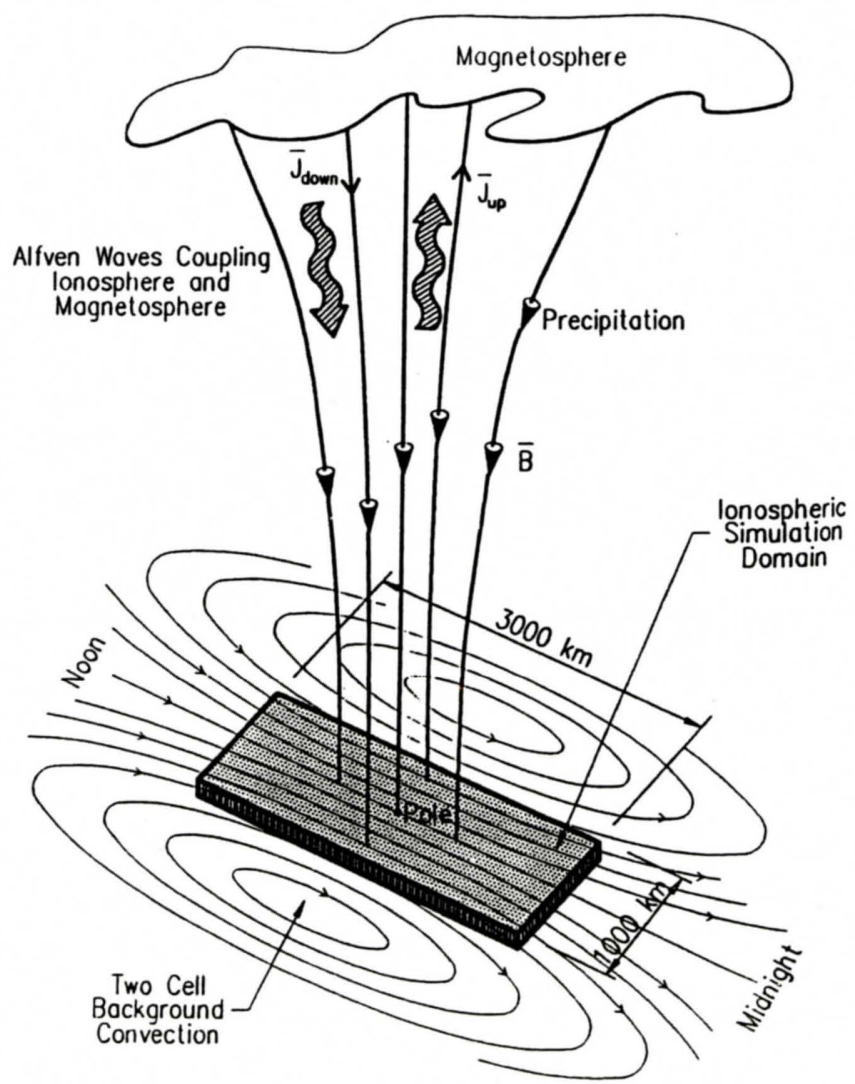

Fig. 1. A schematic diagram showing the geophysical framework of the polar cap arc model. The background ionosphere convection is for illustrative purposes and not necessarily a two-cell convection. 
and the particle precipitation. On the other hand, the existence of mesoscale shear flow associated with polar cap arcs has been confirmed by many observations [Hoffman et al., 1985; Carlson et al., 1988; Mende et al., 1988; Valladares and Carlson, 1991], but the originating mechanism of such a shear flow is unclear at this stage. A natural speculation is that the initial magnetospheric mesoscale convection may already have the shearing feature and that the appearance of polar cap arcs is the consequence of the interactions between this magnetospheric shear flow and the ionosphere. This leads to an important assumption of the model in which we initially assume a mesoscale magnetospheric shear flow carried by a downward propagating Alfvén wave. The question of how this shear flow is originated in the magnetosphere is relevant but beyond the scope of this work. The ionospheric background convection field in the model is the large-scale convection field existing in the ionosphere prior to the arrival of the mesoscale magnetospheric shear flow. It should be noted that the convection electric field of polar cap arcs at an asymptotic steady state is not the simple superposition of the ionospheric background field and the initial mesoscale shear convection field but instead is self-consistently determined by the interactions between the magnetosphere and ionosphere, and it has evolved in a time-dependent dynamical manner.

The physics governing the reflection of upward propagating Alfvén waves at the magnetospheric boundary has been briefly elucidated by Kan et al. [1988]. On open field lines the solar wind inertia is sufficiently large so that the $\mathbf{E} \times \mathbf{B}$ flow of the solar wind is unaffected by the loading of the incident Alfvén wave at the magnetopause. This means that the electric field on open field lines at the magnetopause is kept constant by the solar wind, which can be translated into the condition that the electric field of the reflected wave must cancel the incident wave field, i.e., the reflection coefficient on open field lines at magnetopause is given by $R_{m}=-1$. The situation on closed field lines is quite different. The inertia of the $\mathbf{E} \times \mathbf{B}$ convection in the plasma sheet is extremely limited; therefore, the incident Alfvén wave can be expected to modify the electric field in the plasma sheet on closed field lines. If the inertia in the plasma sheet is sufficiently small, an Alfvén wave incident on the plasma sheet transmits almost without reflection. Consider a pair of identical but oppositely propagating Alfvén waves incident on the plasma sheet simultaneously. They will be fully transmitted in this case. By regarding the transmitted waves as if they were reflected, one can find the equivalent reflection coefficient $R_{m}=+1$. On the other hand, if the inertia in the plasma sheet is sufficiently large such as in the lowlatitude boundary layer, $R_{m}$ may be somewhat less than +1 but larger than -1 . A more recent quantitative study of the reflection of Alfvén waves at the magnetopause has been conducted by Cao and Kan [1990]. By using linearized MHD conservation relations, they confirmed that the reflection coefficient of Alfvén waves on the open magnetopause should be very close to -1 , and the open magnetopause can be treated as a constant voltage source.

The enhancement of the ionospheric conductivity in the model is assumed to be caused mainly by the precipitating electrons associated with the upward field-aligned currents. Maximum flexibility is given to the inputs of the model, which allows us either to simulate polar cap arcs under different conditions or to test various theoretical hypotheses. For example, polar cap arcs in the model can be assumed on open field lines, on closed field lines, or even on both; the large- scale ionospheric background convection can be four-cell, twocell, or even three-cell. A mathematical formulation of the proposed scenario of polar cap arcs is presented in the next section.

\section{Mathematical Formulation}

The ionosphere is treated as a two-dimensional slab with an integrated conductivity. The $\mathrm{X}$ axis measures the noonmidnight dimension and points to the dayside, and the $\mathrm{Y}$ axis measures the dawn-dusk dimension and points to the duskside (see Figure 1). The magnetosphere is a two-dimensional pseudoplane used as a boundary for the reflection of Alfvén waves. The magnetosphere and ionosphere are connected by magnetic field lines which are assumed perpendicular to the ionosphere. The communication between the magnetosphere and ionosphere is characterized by the bouncing of Alfvén waves between these two regions, and both the upward and downward field-aligned currents are associated with the propagating Alfvén waves. Initially, the large-scale ionospheric convection and conductivity are given. These large-scale patterns are consistent with the overall solar, geomagnetic, and IMF conditions. The background convection shown in Figure 1 is not necessarily a two-cell convection; it has only been selected for the purpose of illustration. The ionospheric simulation domain can be on open field lines, on closed field lines, or on both, depending on the choice of the wave reflection character at the magnetospheric boundary. The initial time ( $\mathrm{T}=0 \mathrm{~min}$ ) is the time when the magnetospheric shear flow carried by Alfvén waves first arrives at the ionosphere.

\subsection{Propagation and Reflection of Alfvén Waves}

The propagating Alfvén waves can carry both field-aligned current and transverse polarization current [Kan and Sun, 1985]. The relationship between the wave electric field and the currents can be derived from MHD equations.

For incompressible plasma, the ideal MHD equations can be expressed as

$$
\begin{gathered}
\rho \frac{\partial \mathbf{V}}{\partial t}+\rho(\mathbf{V} \bullet \nabla) \mathbf{V}=\mathbf{J} \times \mathbf{B} \\
\frac{\partial \rho}{\partial t}+\nabla \bullet(\rho \mathbf{V})=0 \\
\mathbf{E}+\mathbf{V} \times \mathbf{B}=0 \\
\nabla \times \mathbf{E}=-\frac{\partial \mathbf{B}}{\partial t} \\
\nabla \times \mathbf{B}=\mu_{o} \mathbf{J}
\end{gathered}
$$

where $\rho$ is the mass density, $\mathbf{V}$ is the fluid velocity, $\mathbf{J}$ is the current density, $\mathbf{B}$ is the magnetic field, $\mathbf{E}$ is the electric field, and $\mu_{O}$ is the free space permeability. The relationship between the fluid velocity $\mathbf{V}$ and the perturbed magnetic field $\mathbf{b}$ of a nonlinear Alfvén wave can be derived from equation (1) [see Alfvén and Falthammar, 1963], which is

$$
\mathbf{V}= \pm \frac{\mathbf{b}}{\left(\mu_{o} \rho_{o}\right)^{1 / 2}}
$$

where $\rho_{O}$ is the background mass density and the plus (minus) sign is for the propagation antiparallel (parallel) to 
background magnetic field $\mathbf{B}_{o}$. The corresponding phase velocities are $\mathbf{V}_{\phi}=\mp \mathbf{V}_{A}$, where $\mathbf{V}_{A}=\mathbf{B}_{o} /\left(\mu_{o} \rho_{o}\right)^{1 / 2}$. Since $\mathbf{b}$ and $\mathbf{V}$ are coplanar, the electric field of an Alfvén wave is

$$
\mathbf{E}=-\mathbf{V} \times \mathbf{B}_{0} .
$$

Inserting equations (2) and (3) into ( $1 e)$, we obtain the relationship between the electric field of an Alfvén wave and the currents carried by the Alfvén wave,

$$
\mathbf{J}_{A}= \pm \Sigma_{A}\left[-\mathbf{b}_{o}(\nabla \cdot \mathbf{E})+\left(\mathbf{b}_{o} \bullet \nabla\right) \mathbf{E}\right]
$$

where $\mathbf{b}_{O}=\mathbf{B}_{o} / B_{o}$ and where $\Sigma_{A}=\left(\mu_{o} V_{A}\right)^{-1}$ is the characteristic conductance of the plasma carrying the Alfvén wave. The first term in equation (4) is the field-aligned current carried by the Alfvén wave, and the second term is the polarization current flowing across field lines along the wave front.

When the Alfvén wave carrying the shear flow arrives at the ionosphere, the wave field can be partially reflected from the ionosphere because of the finite ionospheric conductance. From equation (4), the field-aligned currents carried by the incident and the reflected Alfvén waves can be written as

$$
\mathbf{J}_{\| A}=\mathbf{b}_{o} \sum_{A} \nabla \bullet\left(\mathbf{E}^{i}-\mathbf{E}^{r}\right)
$$

where $\mathbf{E}^{i}$ and $\mathbf{E}^{\boldsymbol{r}}$ are the incident and the reflected Alfvén wave fields, respectively. The current continuity in the ionosphere can be expressed as

$$
\mathbf{J}_{\| i}=\nabla \cdot\left(\Sigma_{P} \mathbf{E}_{i}+\Sigma_{H} \mathbf{b}_{o} \times \mathbf{E}_{i}\right)
$$

and

$$
\mathrm{E}_{i}=\mathrm{E}^{i}+\mathbf{E}^{r}+\mathbf{E}_{i P}
$$

where $\mathbf{E}_{\boldsymbol{i}}$ is the total ionospheric electric field at that moment, $\mathbf{E}_{i P}$ is the ionospheric electric field which excludes the incident and the reflected wave fields, and $\Sigma_{P}$ and $\Sigma_{H}$ are the ionospheric Pedersen conductance and Hall conductance, respectively.

By matching the field-aligned currents carried by the incident and the reflected waves and the field-aligned currents which exist in front of the wave front of the Alfvén waves $\left(J_{\| D}\right)$ with the divergence of the ionospheric currents, we obtain a timedependent equation which describes the reflection of an Alfvén wave from the ionosphere [cf. Zhu and Kan, 1990],

$$
\begin{aligned}
& \Sigma_{A} \nabla \bullet\left[\mathbf{E}^{i}(t)-\mathbf{E}^{r}(t)\right]+J_{I I D}(t)= \\
& \nabla \bullet\left[\Sigma_{P}(t) \mathbf{E}_{i}(t)+\Sigma_{H}(t) \mathbf{b}_{o} \times \mathbf{E}_{i}(t)\right]
\end{aligned}
$$

and

$$
\mathbf{E}_{i}(t)=\mathbf{E}^{i}(t)+\mathbf{E}^{r}(t)+\mathbf{E}_{i P}(t) .
$$

Inspection of equation (7) shows that the reflected Alfvén waves, $\mathbf{E}^{\boldsymbol{r}}$, more precisely referred to as upward propagating Alfvén waves, not only originate from the reflection of the downward propagating Alfvén waves but can also originate from a temporal change of the ionospheric conductance. The latter can be seen clearly if the components of $J_{\| D}$ are written down explicitly, i.e.,

$$
J_{I I D}(t)=\nabla \cdot\left[\Sigma_{P}(t-\Delta t) \mathbf{E}_{i P}(t)+\Sigma_{H}(\mathrm{t}-\Delta t) \mathbf{b}_{o} \times \mathbf{E}_{i P}(t)\right] .
$$

The mechanism of a temporal change of the ionospheric conductance producing an upward propagating Alfvén wave can be briefly elucidated as follows: The temporal change of the ionospheric conductance can lead to an electric field perturbation superimposed on the background ionospheric electric field. The perturbed electric field then leads to the perturbation of magnetic field lines, producing the upward propagating Alfvén waves. The significance of the upward propagating Alfvén waves due to the temporal change of the ionospheric conductance in equation (7) is that it shows the ionosphere plays an active role in the M-I coupling system. The upward propagating Alfvén waves continuously send updated information about changes in the ionosphere to the magnetosphere, thereby keeping the interaction between the magnetosphere and the ionosphere internally consistent.

On the magnetospheric side, a reflection coefficient is used to describe the reflection of the upward traveling Alfvén waves at the magnetospheric boundaries [cf. Kan et al., 1988], which can be expressed as

$$
\begin{gathered}
R_{m}=-1 \text { on open field lines } \\
-1<R_{m}<1 \text { on closed field lines. }
\end{gathered}
$$

The physics governing the reflection coefficient has been discussed in the previous section. It may be noted that $R_{m}=-1$ corresponds to an idealized constant voltage source while $R_{m}=$ 1 corresponds to a constant current source. The value of $R_{m}$ on closed field lines can be expected to increase from slightly greater than -1 toward +1 as one moves from the distant tail or the low-latitude boundary layer toward the inner edge of the plasma sheet. The traveling time of Alfvén waves, which is defined as the time for the propagating Alfvén waves to finish a round trip between the magnetosphere and the ionosphere, can vary from about $1 \mathrm{~min}$ to about $10 \mathrm{~min}$ depending on which region in the magnetosphere the field lines are connected with.

\subsection{Conductivity Enhancement in the Ionosphere}

Under the assumption that precipitating electrons associated with upward field-aligned currents are the main external ionization source in the polar cap in addition to solar UV contributions, the time-dependent electron continuity equation can be used to describe the ionization in the ionosphere. We have

$$
\frac{\partial N}{\partial t}+\nabla \cdot(N V)=-\frac{Q J_{1}}{e}-\frac{J_{1}}{e H}+\mathrm{S}_{o}-\beta N^{2}
$$

where $N$ is the electron number density in the ionosphere, $Q$ is the ionization coefficient which will be defined and discussed later in this section, $H$ is the effective thickness of the conducting ionosphere, $\beta$ is the recombination coefficient, and $S_{o}$ is the background ionization source term which can be determined by the background convection $\mathbf{V}_{O}$ and background electron density $N_{O}$, i.e.,

$$
\mathrm{S}_{o}=\nabla \cdot\left(N_{o} \mathbf{v}_{o}\right)+\beta N_{o}^{2} .
$$

Please note that the first two terms on the right side of equation (10) are the ionization terms associated with the field-aligned currents, in which the upward current has a negative value. 
From the definition of the Hall conductivity, the heightintegrated Hall conductivity in the $E$ layer of the ionosphere can be approximated by

$$
\Sigma_{H}=\frac{N e H}{\mathbf{B}_{o}} .
$$

By using equation (12), and assuming that the ratio $R=\Sigma_{H} / \Sigma_{P}$ is independent of the energy of the precipitating electrons, equation (10) can be rewritten as

$$
\begin{gathered}
\frac{\partial \Sigma_{H}}{\partial t}=-\frac{(Q H+1)}{B_{o}} \nabla \cdot\left(\Sigma_{P} \mathbf{E}_{i}+\Sigma_{H} \mathbf{b}_{o} \times \mathbf{E}_{i}\right)+ \\
\frac{e H}{B_{o}} \mathrm{~S}_{o}-\frac{\beta B_{o}}{e H} \Sigma_{H}^{2}+\nabla \Sigma_{H} \cdot \frac{\mathbf{b}_{o} \times \mathbf{E}_{i}}{B_{o}} .
\end{gathered}
$$

In this model, the ratio $R$ is assumed to be 1.5 . It should be pointed out that in reality, the ratio between the Hall conductance and the Pedersen conductance increases as the characteristic energy of precipitating electrons increases. Therefore, the adoption of a constant $R$ in the model is a first approximation and can be removed in further improvement of the model, even though a constant $R$ is somewhat acceptable in the polar cap owing to the softer energy spectrum of precipitating electrons compared to that in the auroral oval regions.

The introduction of the ionization coefficient in the electron continuity equation was initially for the treatment of the ionization caused by auroral precipitation in the oval regions [cf. Kan et al., 1988; Zhu and Kan, 1990]. It has been found by many researchers [e.g., Akasofu and Roederer, 1983; Frank et al., 1986] that the overall character of the polar cap arcs is very similar (with respect to electron precipitation and field-aligned currents) to that of arcs in the auroral oval. The key physics contained in the ionization coefficient is the approximately linear $J_{\|}-\phi_{\|}$relationship [Fridman and Lemaire, 1980]. In this model, we assume that such a linear $J_{\|}-\phi_{\|}$relationship can be applied to the acceleration regions of precipitating electrons associated with polar cap arcs; therefore, the proposed ionization coefficient $Q$ [cf. Zhu and Kan, 1990] can be used in this model, which is defined as

$$
\begin{aligned}
& Q=0 \quad J_{\|} \text {downward or } J_{\|} \mid<J_{o} \\
& Q=Q_{o} \quad J_{\|} \text {upward and } \nabla \cdot \mathbf{E}_{i} \geq 0 \text { and } J_{\|} \mid>J_{o} \\
& Q=\gamma^{\prime}\left(J_{\|} \mid-J_{0}\right) J_{\|} \text {upward and } \nabla \cdot \mathbf{E}_{i}<0 \text { and } J_{\|} \mid>J_{o}
\end{aligned}
$$

where $Q_{o}$ is a constant depending on the energy of precipitating electrons which are not associated with potential drop structures along magnetic field lines, $\gamma^{\prime}$ is a parameter which depends on the $J_{\|}-\phi_{\|}$relationship [Fridman and Lemaire, 1980], and $J_{O}$ is the thermal electron current in the loss cone. The ionization coefficient, $Q$, is defined as the average number of ions produced by an incident electron per meter which is proportional to the energy of the precipitating electrons. Since the enhancement of ionospheric conductance is assumed to be caused exclusively by the precipitating electrons, $Q$ should be zero in the regions where the fieldaligned currents are downward. In the regions where $J_{\|}$is upward and $\nabla \cdot \mathbf{E}_{i}>0$, the potential drop structures do not exist along magnetic field lines [Lyons, 1980]. Therefore, the energy of the precipitation in these regions is directly determined by the electron thermal energy in the magnetospheric source regions, and we assume $Q$ to be constant. In the regions of polar cap arcs where $\nabla \cdot \mathbf{E}_{i}<0$, the precipitating electrons are accelerated by field-aligned potential drop. Since the upward field-aligned current density $J_{\|}$linearly depends on the potential drop $\phi_{\|}$along magnetic field lines, the energy of precipitating electrons can be considered to be linearly proportional to the upward fieldaligned current density in these regions, and $Q$ can no longer be a constant, as shown in equation (14). The linear relationship between the ionization coefficient, $Q$, and field-aligned current density in equation (14) means that the characteristic energy of precipitating electrons is, in our model, a temporal and spatial variable determined by the field-aligned current distribution. It should be pointed out that in the present model, we only include the effects of the field-aligned potential drops on the ionization in the ionosphere and have not considered the interactions between Alfvén waves and field-aligned potential drops. Goertz [1984] has used the theory of kinetic Alfvén waves to study the evolution of nonstationary field-aligned potential drops. An improvement of the model along this line can be worthwhile.

Equations (7), (9), (11), (13), and (14) form the basic working equations of the present model of polar cap arcs. The temporal evolution of polar cap arcs and Alfvén waves propagating along magnetic field lines can be obtained by solving these equations numerically.

\subsection{Numerical Scheme of the Model}

The ionospheric simulation domain is $3000 \mathrm{~km}$ long in the $\mathrm{X}$ (midnight-noon) direction and $1000 \mathrm{~km}$ wide in the $\mathrm{Y}$ (dawndusk) direction. The grid size is $30 \mathrm{~km}$ in the $\mathrm{X}$ direction and $10 \mathrm{~km}$ in the $\mathrm{Y}$ direction. The third dimension along the magnetic field lines is a pseudodimension which merely serves to provide the Alfvén wave traveling time scale. The magnetosphere is simplified as a plane which is the source region of the initial magnetospheric shear flow and is used only for treating the reflection of the Alfvén waves on the magnetospheric side after $\mathrm{T}=0 \mathrm{~min}$. The choice of boundary conditions in the simulation is based on the following physical considerations: The polar cap arcs are mainly aligned in the noon-midnight direction. Typically, polar cap arcs can be a few ten to a few hundred kilometers wide and extremely stretched (a few hundred to a few thousand kilometers) in the noon-midnight direction, sometimes even connecting both the dayside and the nightside of the auroral oval. In the distant regions on the morningside or duskside of the polar cap arcs, the background ionosphere can be assumed undisturbed; therefore, we use a constant boundary condition for the dawnside and duskside boundaries in the simulation. On the noonside and midnightside boundaries, the situations are completely different. Not only do the elongated polar cap arcs reach or even go beyond the boundaries into the auroral oval, but also the large-scale convection which is mainly in the Sunaligned direction can carry the disturbance associated with the polar cap arcs through the boundaries. Accordingly, we use an open boundary condition for the noonside and midnightside boundaries, which allows plasma to freely flow through.

Figure 2 illustrates the numerical procedure of our computer model in which the elements in the box of dashed lines are the time-dependent components of the model. In terms of the 


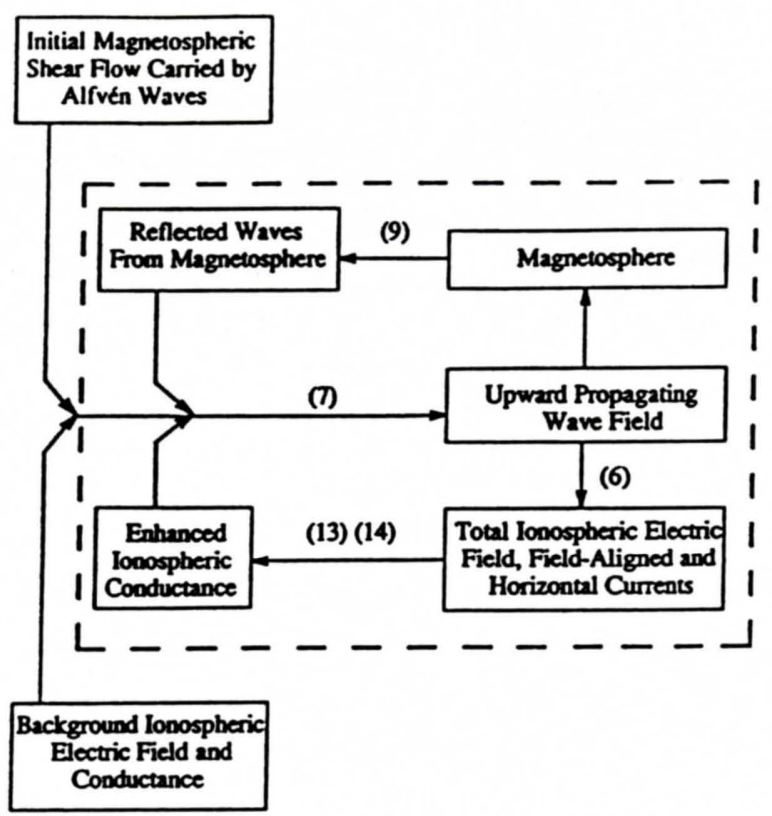

Fig. 2. A flow chart showing the numencal procedure of the polar cap arc model.

diagram, the numerical procedure can be summarized as follows:

1. Starting from the left of the diagram, the initial magnetospheric shear flow carried by the Alfvén waves, background ionospheric convection, and background ionospheric conductance are given first.

2. By using the given downward propagating waves and background ionospheric electric field and conductance, the upward propagating wave field from the ionosphere $\left(\mathbf{E}^{r}\right)$ can be determined by the numerical solution of equation (7).

3. Then calculate the total ionospheric electric field, fieldaligned current, and horizontal ionospheric current at that moment by using equation (6).

4. The reflected wave from the magnetosphere can be determined by equation (9), which contains a time delay depending on the Alfvén wave traveling time. The reflected wave from the magnetosphere will be used as the incident wave for the ionosphere in the later time steps.

5. At the same time, the conductance in the ionosphere increases as a result of the precipitating electrons carried by upward field-aligned currents. The time scale of this ionization process is on the order of the recombination time scale. The enhancement of the ionospheric conductance is determined by equations (13) and (14).

6. We go back to equation (7) to determine the upward propagating Alfvén waves of the next time step by using the reflected waves from the magnetosphere and the newly calculated ionospheric electric field and conductance. Note that even though there is no incident wave from the magnetosphere, the upward propagating waves are not necessarily equal to zero because the ionospheric conductance keeps changing and so can launch upward propagating Alfvén waves.

Because of finite conductance in the ionosphere, the temporal changes of the Alfvén waves along magnetic field lines diminish, and therefore the development of the polar cap arcs and the whole M-I system will eventually approach an asymptotic steady state. Repeating steps 3 to 7 , the ionospheric convection, field-aligned current, ionospheric conductance, integrated horizontal current, and Joule heating rate will change self-consistently with time, and the temporal evolution and spatial structures of the polar cap arcs can be examined in detail.

\section{Inttial StUdy: A CASE RuN}

The observed features of polar cap arcs have a great diversity and sometimes are in contradiction. A theoretical modeling effort should not try to fit itself into a specific observational frame. Instead, a theoretical effort should be made on the basis of the most generalized observational features, and the modeling should be performed in the most general and simplest way. Based on this viewpoint, we make the initial conditions of our modeling as simple and general as possible in order to reveal the basic physics of polar cap arcs. In this paper, we specifically study the features of polar cap arcs which occur on open field lines and are associated with large-scale antisunward background convection in the ionosphere. Figure 3 shows the ionospheric background conditions, and Figure 4 shows the initial perturbation of the magnetosphere-originated shear flow carried by downward propagating Alfvén waves. The top panel of Figure 3 shows the Hall conductance distribution in the noon-midnight cross-section. In the dawn-dusk direction, we assume the Hall conductance to be uniform. This background ionospheric conductance is merely due to the solar contribution, and for simplicity, we assume it decreases linearly toward the nightside. The solid line in the bottom panel of Figure 3 shows the background ionospheric potential distribution in the dawn-dusk cross-section. In the noonmidnight direction, the electrical potential is constant. For comparison purposes, the potential perturbation associated

IONOSPHERIC BACKGROUND
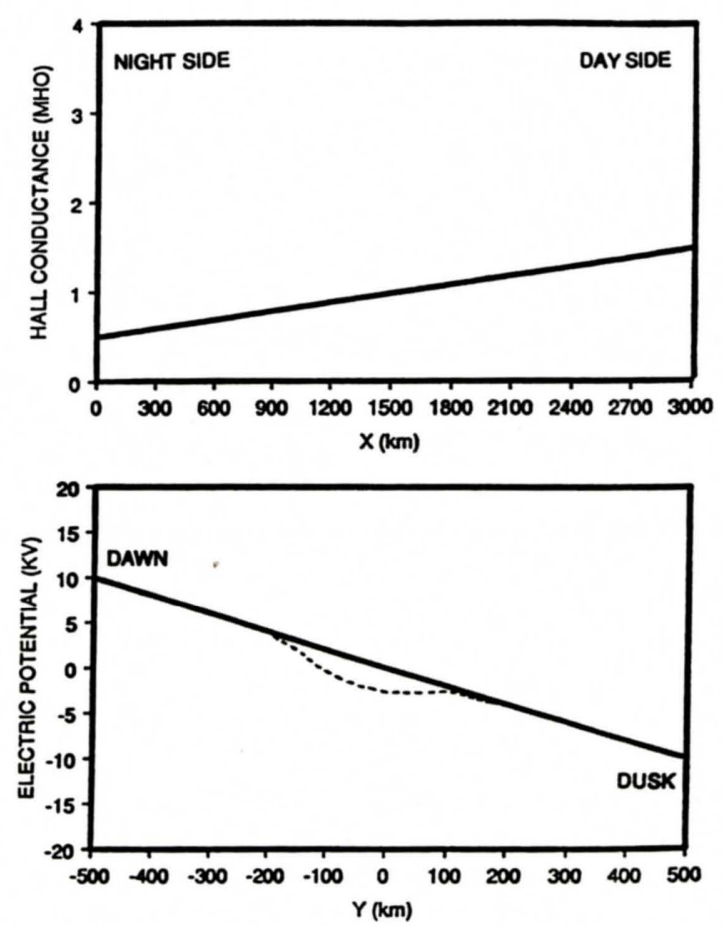

Fig. 3. Ionospheric background conditions. (Top) The Hall conductance distribution in the noon-midnight cross-section. (Bottom) The ionospheric background potential distribution in the dawn-dusk cross-section in which the dashed line represents the potential perturbation associated with the initial magnetospheric shear flow. 
INITIAL PERTURBATION

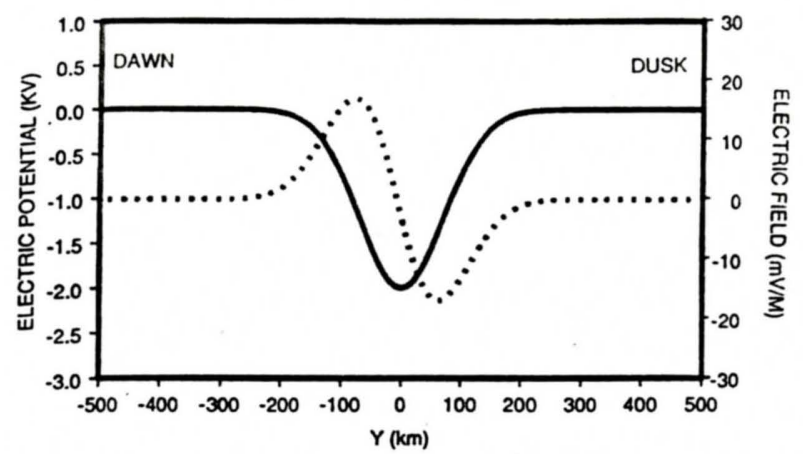

Fig. 4. Potential distribution in the dawn-dusk cross-section associated with the initial magnetospheric shear flow (solid line) and the corresponding electric field distribution (dashed line).

with the initial magnetospheric shear flow is shown by the dashed line in the panel. It can be seen that the distribution of the background ionospheric potential in the bottom panel of Figure 3 implies a large-scale antisunward convection which is uniform in the dawn-dusk direction. The initial magnetospheric shear flow carried by the propagating Alfvén waves is represented by a potential in the Gaussian distribution as shown by the solid line in Figure 4 . The shear flow is uniform in the noon-midnight direction. The corresponding electric field distribution, which is convergent in the center and divergent on the edges of the shear flow, is shown by the dashed line in the same figure.

Since we have assumed that polar cap arcs occur on open field lines in this specific case, the reflection coefficient at the magnetospheric boundary shown in equation (9) is -1 everywhere in the calculation domain. A constant ratio between the Hall conductance and the Pedersen conductance $R=$ 1.5 is adopted as an approximate treatment. The time step is $10 \mathrm{~s}$, and the Alfvern wave traveling time between the magnetospheric boundary and ionosphere is assumed to be 2 $\mathrm{min}$. Other model parameters are as follows: thermal electron current $J_{O}=0.08 \mu \mathrm{A} \mathrm{m}^{-2}$, constant ionization coefficient $Q_{o}=$ $7 \times 10^{-3}$ ions per electron per meter, coefficient $\gamma^{\prime}=1 \times 10^{4}$ (ions m)/(electron A) [Rees, 1963; Fridman and Lemaire, $1980]$, and recombination coefficient $\beta=0.9 \times 10^{-13} \mathrm{~m}^{3} \mathrm{~s}^{-1}$ [Walls and Dunn, 1974].

\subsection{Polar Cap Arcs at Asymptotic Steady State}

The initial shear flow carried by Alfvén waves arrives in the ionosphere at $\mathrm{T}=0 \mathrm{~min}$, which leads to the bouncing of Alfvén waves between the magnetosphere and ionosphere and the development of polar cap arcs. As long as the external environment of the coupled M-I system remains unchanged, the bouncing of Alfvén waves eventually will be damped by the finite ionospheric conductance, and the development of the polar cap arcs will approach an asymptotic steady state. In this case, such a steady state of the polar cap arcs is achieved in about 8 to $10 \mathrm{~min}$. Figures 5 to 8 show the asymptotic features of the polar cap arcs at time $T=8 \mathrm{~min}$. Figure 5 shows the field-aligned current distribution in which the dashed lines denote upward field-aligned currents and the solid lines denote downward field-aligned currents. Please note that only a portion of the simulation domain is shown in this figure in order to display the detailed structures associated with the polar cap arcs. It can be seen, as indicated by Figure 5, that in the

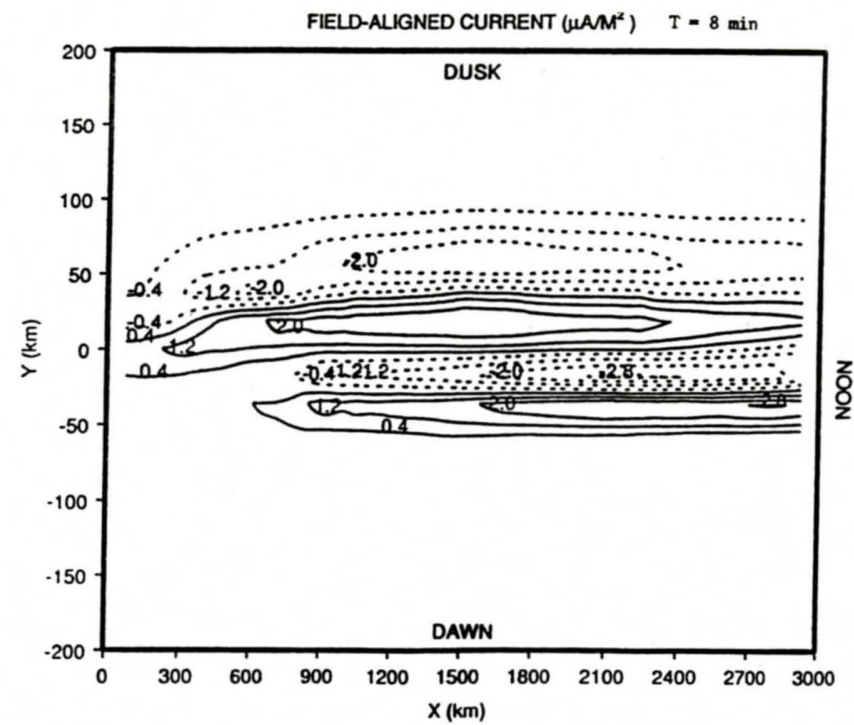

Fig. 5. Field-aligned current distribution at the asymptotic steady state in which the dashed lines denote the upward currents and the solid lines denote the downward currents.

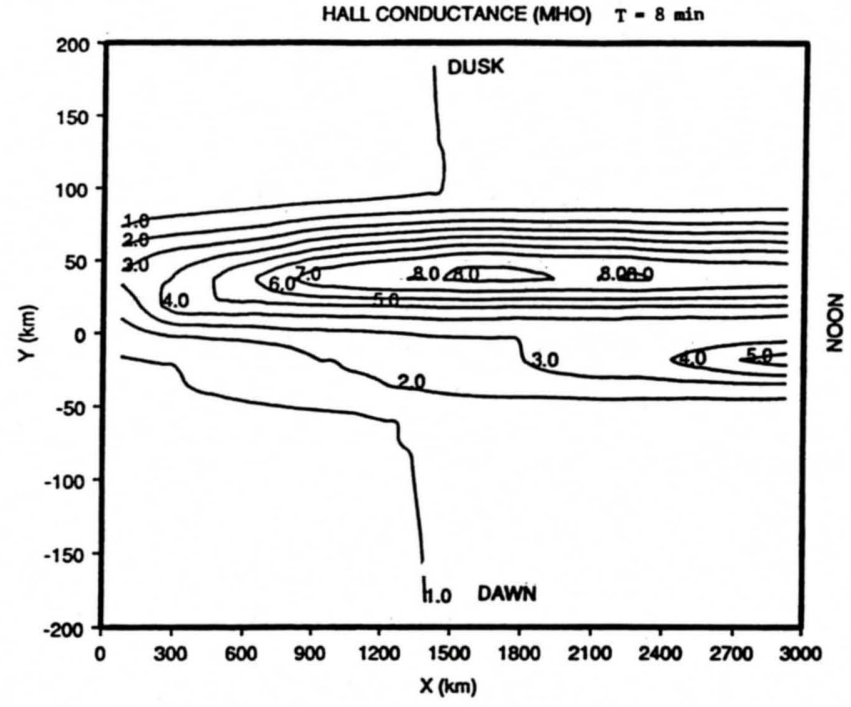

Fig. 6. Hall conductance distribution at the asymptotic steady state.

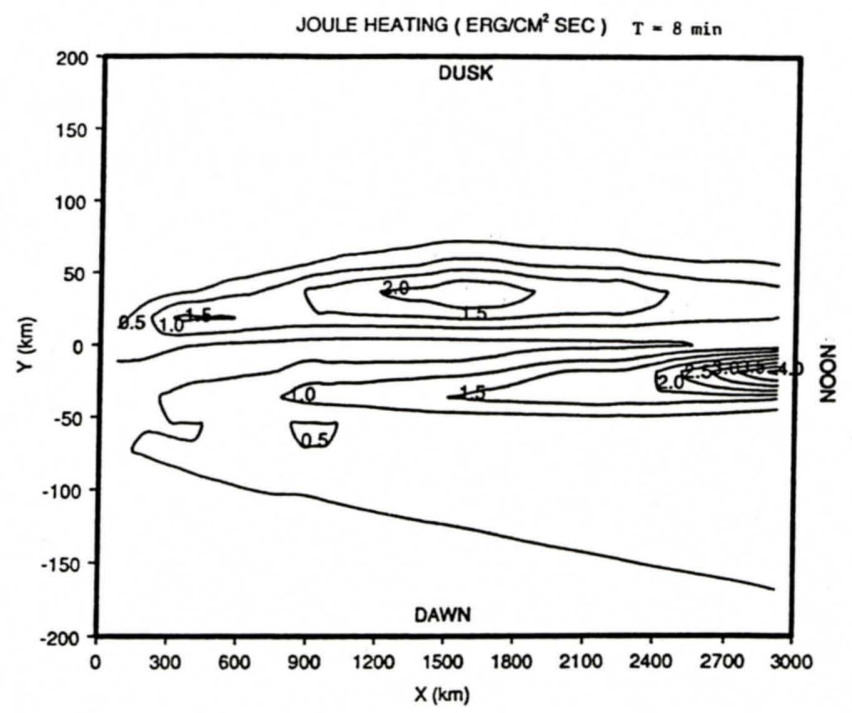

Fig. 7. Distribution of Joule heating rate at the asymptotic steady state. 
$T=8 \mathrm{~min} . \quad X=1500 \mathrm{~km}$
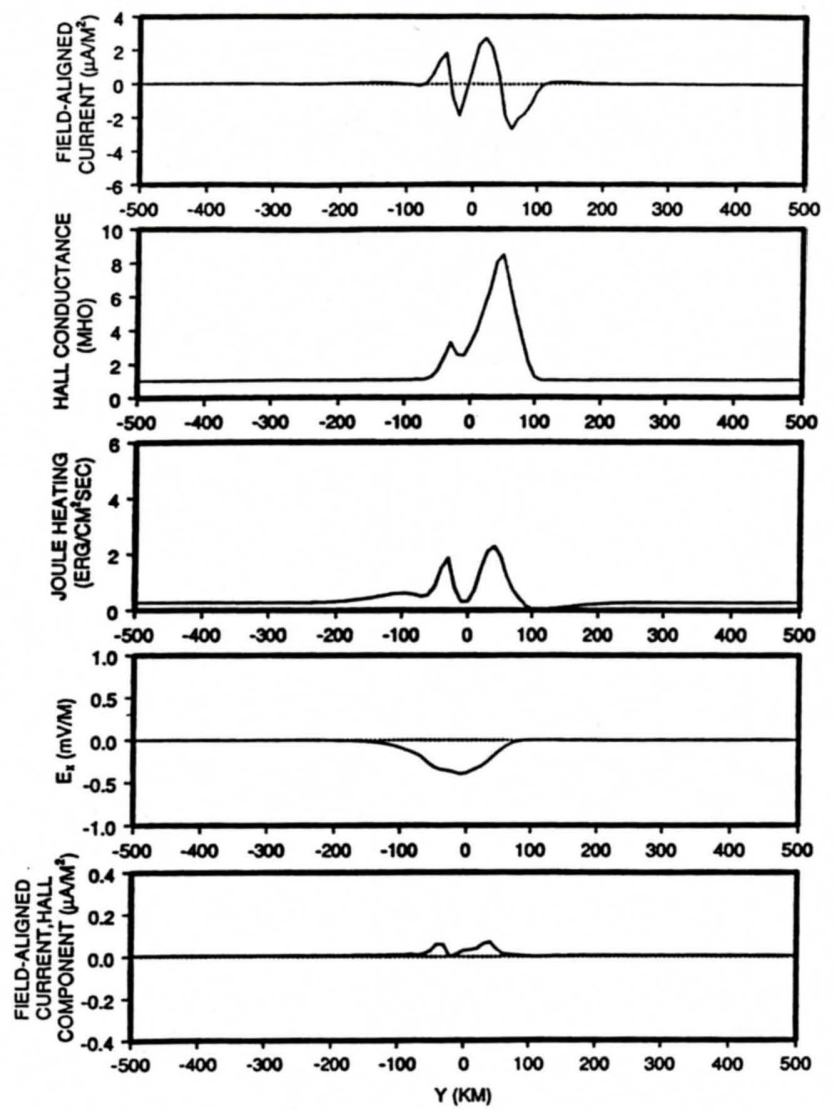

Fig. 8. Asymptotic distributions of the field-aligned currents, Hall conductance, Joule heating rate, day-night component of the electric field, and the Hall component of the field-aligned currents along the dawn-dusk cross-section $(x=1500 \mathrm{~km})$.

self-consistent development of a polar cap arc, a single-peak precipitation associated with the initial magnetospheric shear flow has been split into multiple peaks, leading to multiple structures of the polar cap arcs. An individual arc is about 30 to $40 \mathrm{~km}$ wide and is separated by a downward current region. The observed multiple polar cap arcs are not due to any multiple structures in the magnetospheric source region; instead, such a multiple feature is internally originated in the dynamical process of the polar cap arcs determined by the whole coupled M-I system. Figure 5 shows that strong downward field-aligned currents can develop near the most intensive upward fieldaligned currents and form pair structures of field-aligned currents. The spatial scale of this kind of pair structure in this case is around tens of kilometers with a downward current on the morningside. The maximum intensities of both upward and downward currents shown in Figure 5 are about $2.8 \mu \mathrm{A} / \mathrm{m}^{2}$. In this case, the polar cap arcs are embedded in a weak large-scale downward field-aligned current extending from the vicinity of the arcs to the dawnside and duskside boundaries.

Figure 6 shows the distribution of the ionospheric Hall conductance at the asymptotic steady state. In the regions of the polar cap arcs, the ionospheric conductance is greatly enhanced by the precipitating electrons carried by upward currents, while the conductance in the regions outside of the polar cap arcs remains almost unchanged. In this case, the maximum increase of the Hall conductance in the polar cap arcs is about 6 mhos. Like the polar cap arcs, the enhanced conductance is also aligned in the noon-midnight direction.
The asymptotic distribution of the Joule heating rate is shown in Figure 7. A very sharp change of the Joule heating rate can be seen in the polar cap arcs compared to that in the regions outside of the arcs. The maximum Joule heating rate in this case is around $5.4 \mathrm{ergs} / \mathrm{cm}^{2} \mathrm{~s}$. Comparing Figures 5, 6, and 7, it is seen that the regions of strong ionization coincide with the regions of intensive upward field-aligned currents, but the regions of strong Joule heating appear to have a dawnward shift to the region of the intensive upward currents.

To examine more detailed spatial features of the polar cap arcs, the distributions of the field-aligned currents, Hall conductance, Joule heating, day-night component of the electric field, and the Hall component of the field-aligned currents along the dawn-dusk cross-section $(x=1500 \mathrm{~km})$ are shown in Figure 8. A clear pattern of the multiple structure of the polar cap arcs can be seen in the top panel where the negative value is for an upward current. The multiple peaks of conductance enhancement are due to the multiple structure of the precipitation associated with field-aligned currents. The maximum Joule heating does not occur in the regions of maximum conductivity enhancement or maximum precipitation; instead, it shifts about $30 \mathrm{~km}$ toward the morningside. In the region of maximum precipitation, Joule heating has a moderate value. This result can be explained by the dramatic decrease of the electric field due to the conductivity enhancement in the regions of strong precipitation. Whether the plasma flow crossing the polar cap arcs, which is initially zero in our modeling, exists or not is an important issue. Our results do show the existence of such a flow, which is associated with the $\mathrm{X}$ component of the electric field in our modeling, when the polar cap arcs are fully developed, but the amplitude of it is very small, as shown in Figure 8, and the characteristic time scale of it is much larger than the time constant for the polar cap arcs to develop. Therefore, the effect of plasma flow crossing the polar cap arcs is not significant in this case. Another interesting feature shown in Figure 8 is the closure of field-aligned current in the ionosphere. It is known that a field-aligned current can be closed by the ionospheric Hall current or Pedersen current. It has been found [Kan et al., 1988] that in the auroral oval regions, a significant amount of the field-aligned current associated with bright arcs is connected to the ionospheric Hall current and the divergences of the Hall and Pedersen currents have the same sign in these regions. Our results show that the features of current closure in polar cap arcs are different from those of the arcs in auroral oval regions. First, the contribution of the Hall component of fieldaligned current is not significant in polar cap arcs, as shown in Figure 8 , and the field-aligned current is mainly closed by the Pedersen current. Second, the divergences of the Hall and Pedersen currents are in opposite sense in polar cap arcs, which means the Hall and Pedersen components of field-aligned current cancel each other, which is the opposite of the effect seen in the auroral oval regions.

\subsection{Evolution of Polar Cap Arcs}

Figure 9 shows the snapshots of the evolution of polar cap arcs. The top panels of Figure 9 show the field-aligned current distributions at $\mathrm{T}=3 \mathrm{~min}$ and $\mathrm{T}=6 \mathrm{~min}$, the middle panels show Hall conductance distributions, and the bottom panels show Joule heating rate distributions. This result clearly shows that the polar cap arcs at the steady state shown in Figures 5 to 8 do undergo a temporal evolution determined by a 
FIELD-NLIGNED CURRENT ( $\left.M M M^{2}\right) \quad T=3$ ain

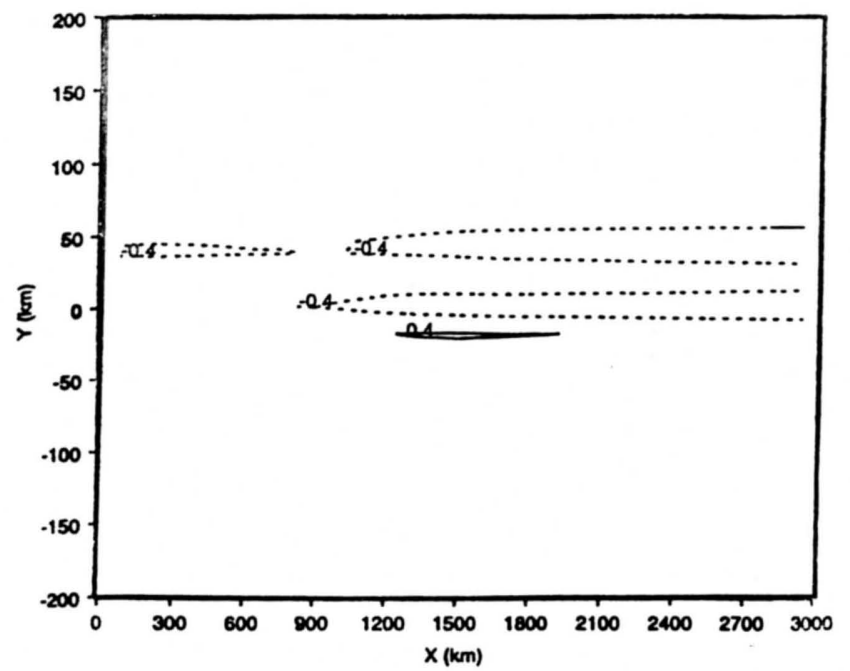

HALL CONOUCTANCE (MHO) T $\quad 3$ min

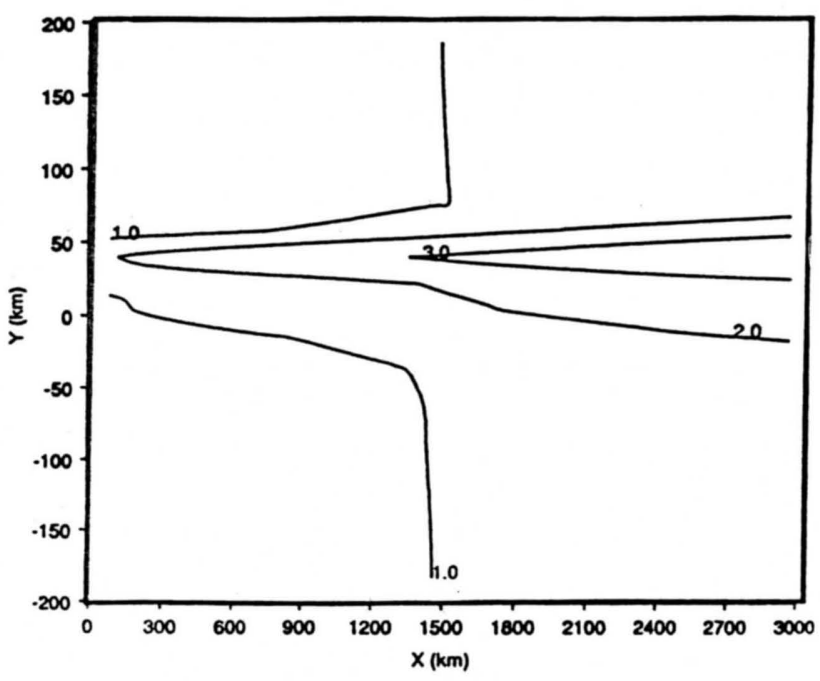

JOULE HEATING (ERGCAR SEC) T - 3 an

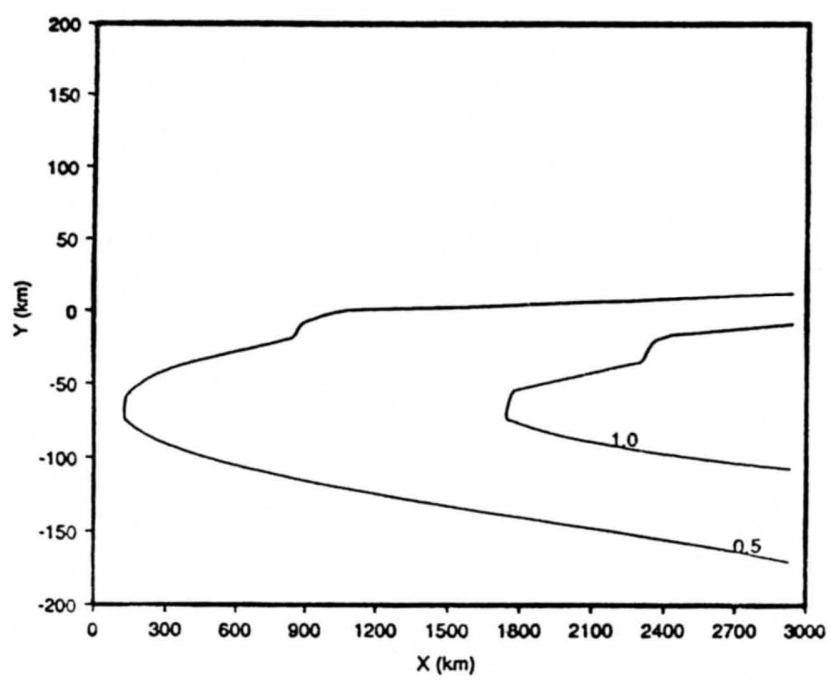

FIELD-ALIGNED CURRENT ( $\left.M M M^{2}\right) \quad T$ - 6 win

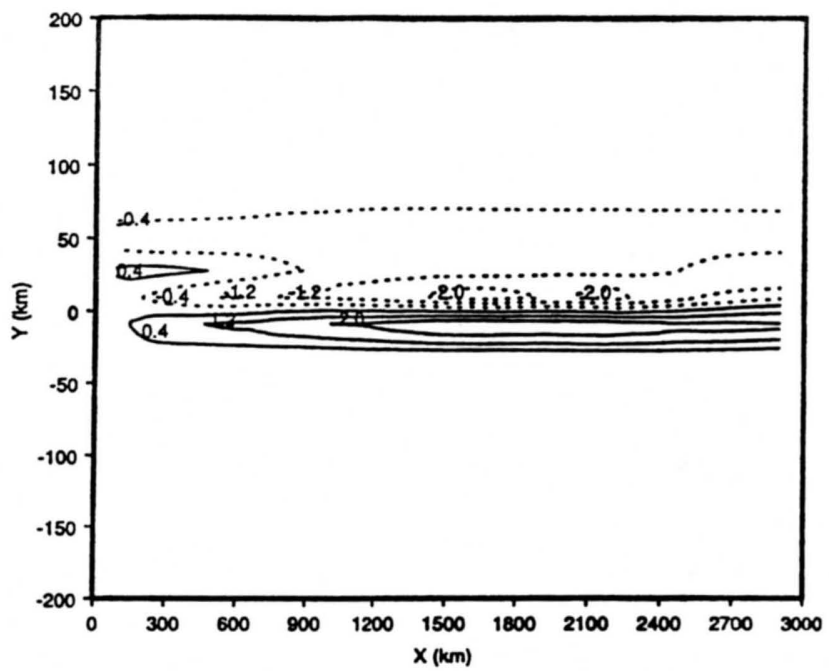

HNL CONDUCTANCE (MHO) T $\quad \mathrm{T}$ ain

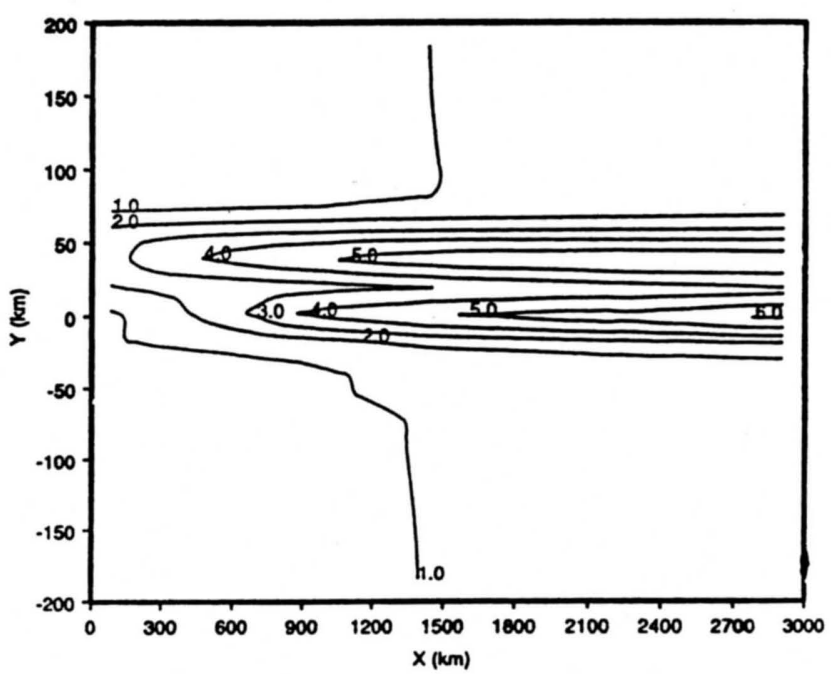

JOULE HEATING (ERGCMa SEC) T - 6 aln

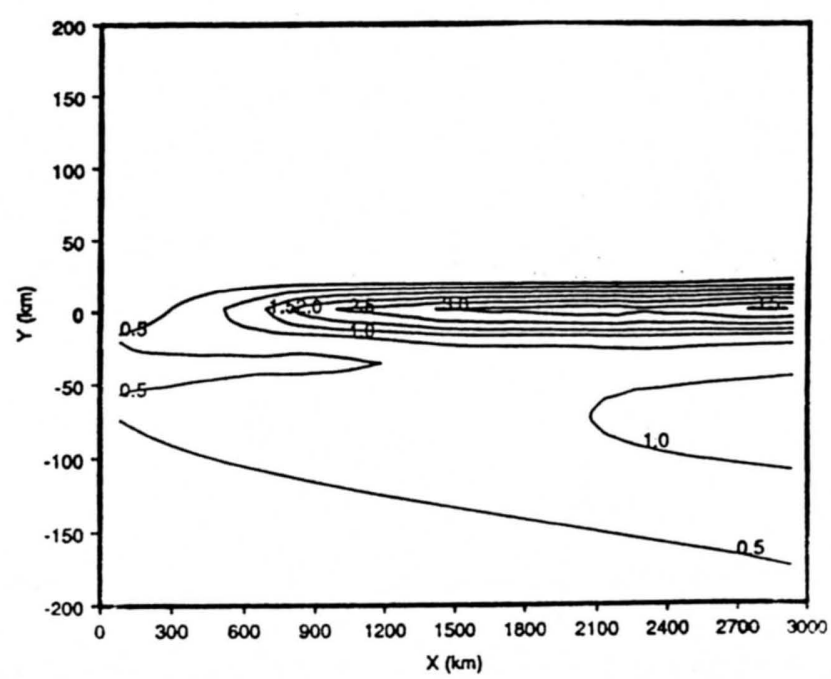

Fig. 9. Snapshots of the temporal evolution of (top) the field-aligned currents, (middle) Hall conductance, and (bottom) Joule heating rate. 
dynamical process, and they are not the simple superposition of the ionospheric background and imposed magnetospheric shear flow. Figure 10 shows the temporal evolution of the field-aligned current distribution along the dawn-dusk crosssection $(x=1500 \mathrm{~km})$. It can be seen that individual arcs are not spatially stable in time and they move at an average velocity of around $200 \mathrm{~m} / \mathrm{s}$ while their intensities increase. Multiple structures appear very early in the development stage of the arcs, but different arcs are not enhanced evenly in time. The breakup of a single arc is seen first, and a few minutes later, the breakup of a second arc occurs at a distance of tens of kilometers away from the first one. Figures 11 and 12 show the snapshots of the evolution of the Hall conductance distributions and Joule heating distributions along the dawndusk cross-section $(x=1500 \mathrm{~km})$. These two quantities change self-consistently while the polar cap arcs are developing.

Figure 13 shows the evolution of the total upward and downward field-aligned current in the region which covers the range from $\mathrm{y}=-200 \mathrm{~km}$ to $\mathrm{y}=200 \mathrm{~km}$ in the dawn-dusk direction and from $\mathrm{x}=0 \mathrm{~km}$ to $\mathrm{x}=3000 \mathrm{~km}$ in the noonmidnight direction. As expected, both total upward and downward currents in this region increase in time while the polar cap arcs are developing. One interesting thing shown in Figure 13 is the temporal variation of the difference between the total upward and total downward currents. In the early development stage of polar cap arcs, a substantial difference exists between the total upward and total downward currents. This means that a significant amount of upward current in the arcs connects to distant ionospheric horizontal currents outside of the regions of the polar cap arcs. While the polar cap arcs are developing, the conductance in the regions of the polar cap arcs is enhanced, and less current flows into the darkened regions outside of the polar cap arcs; thereby more upward current is closed by local downward current, and the total downward current in the region catches up gradually. At the asymptotic steady state of the arcs, the difference between the total upward and total downward currents eventually approaches zero. This indicates that while polar cap arcs are developing, the associated current system tends to become further localized, and at steady state the current system in the narrow regions around the arcs is locally self-closed.
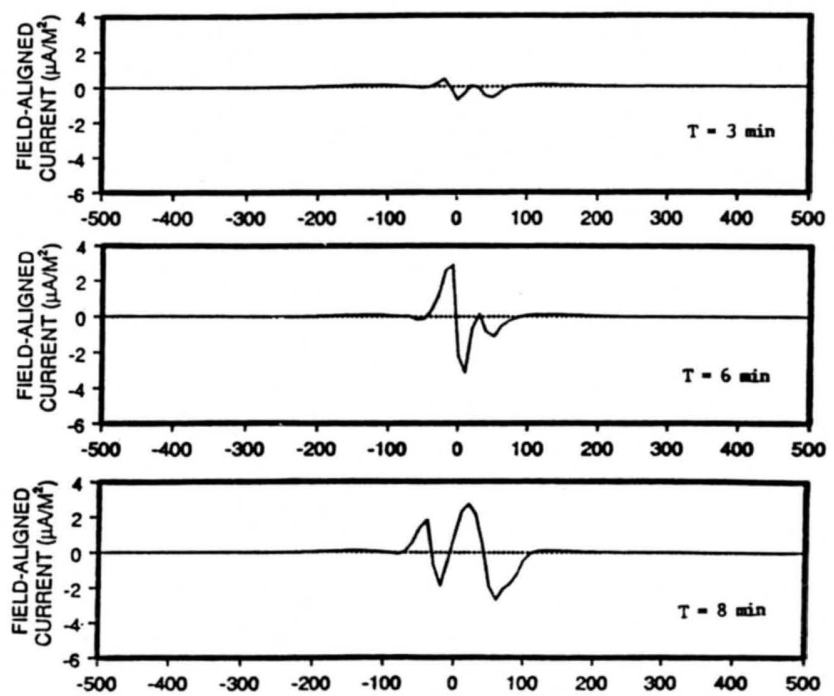

Fig. 10. Snapshots of the temporal evolution of the field-aligned current distribution in the dawn-dusk cross-section $(x=1500 \mathrm{~km})$.
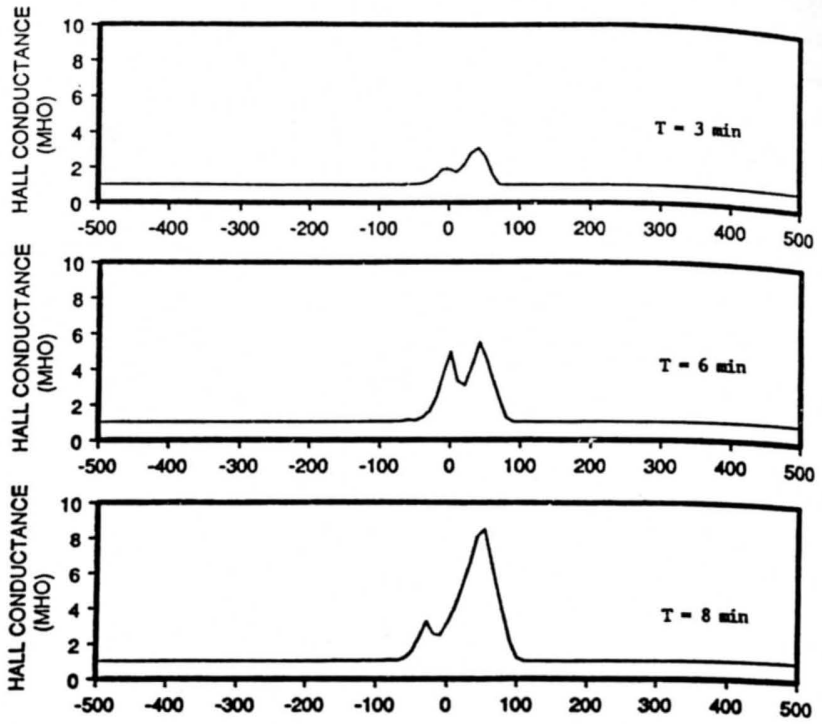

Fig. 11. Snapshots of the temporal evolution of the Hall conductance distribution in the dawn-dusk cross-section $(x=1500 \mathrm{~km})$.
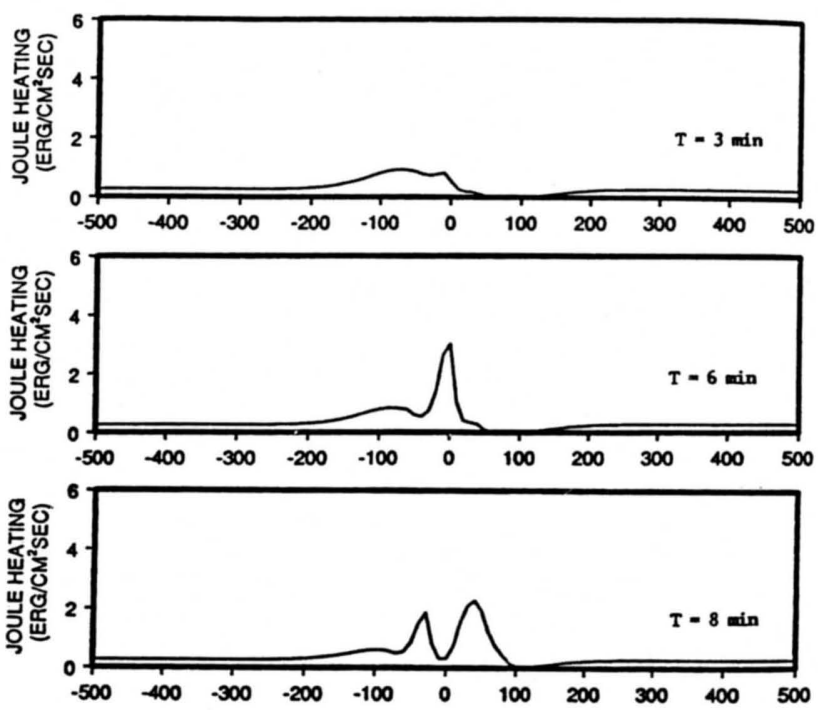

Fig. 12. Snapshots of the temporal evolution of the Joule heating rate distribution in the dawn-dusk cross-section $(x=1500 \mathrm{~km})$.

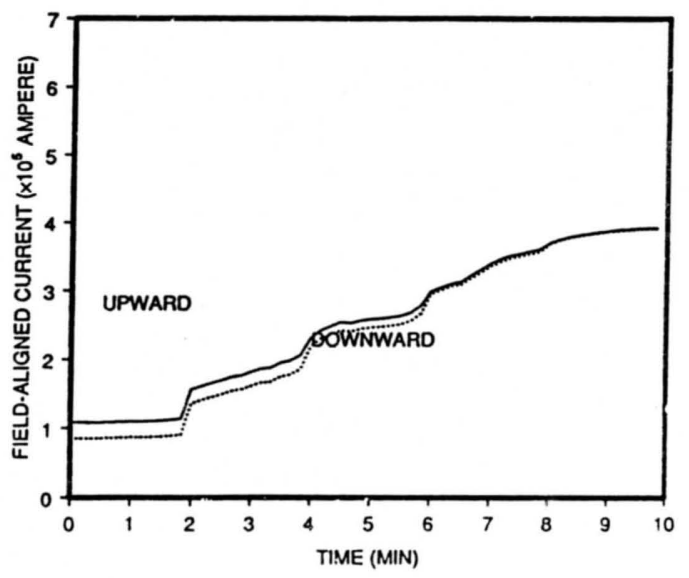

Fig. 13. Temporal evolution of the total upward and downward fieldaligned currents in the region which covers the range from $y=-200 \mathrm{~km}$ to $y=200 \mathrm{~km}$ in the dawn-dusk direction and from $\mathrm{x}=0$ to $\mathrm{x}=3000 \mathrm{~km}$ in the noon-midnight direction. 
Figure 14 shows the evolution of the ratio between the total Joule heating and the total energy flux associated with precipitating electrons in the region around the polar cap arcs as described above. Initially, the ratio is about 8.2. When the polar cap arcs are fully developed, the ratio drops to around 0.4 . A commonly accepted fact is that in the polar cap, the Joule heating rate is much larger than the energy deposition from the precipitating electrons. But our results suggest that this might not be true in the small-scale region around the polar cap arcs. A ratio of less than 1 can exist when the electric field is dramatically decreased and precipitation is greatly enhanced in the narrow regions around the arcs.

\section{SUMMARY}

A quantitative time-dependent model of polar cap arcs has been developed in which the electrodynamics of the polar cap arcs are treated self-consistently in the frame of the coupled magnetosphere-ionosphere system. The goal of developing such a quantitative model is to understand the basic physics of polar cap arcs and provide a theoretical guide for synthesizing the diversified observational results. The model specifically focuses on the features of mesoscale spatial structures and temporal evolution of polar cap arcs rather than the associated large-scale ionospheric background and their magnetospheric connections. To reveal the most basic physics of the arcs, the model has been formulated to be as simple and general as possible, but contains key physical processes. A maximum flexibility has been given to the inputs of the model, which allows us either to simulate various polar cap arcs under different conditions or to test various theoretical hypotheses in some aspects of the model. An initial study of the polar cap arcs has been done by using the developed model in which the polar cap arcs are assumed to occur on open field lines and to be associated with large-scale antisunward background convection in the ionosphere. The main results of the initial study can be summarized as follows:

1. The time constant for the formation of the polar cap arcs is around $10 \mathrm{~min}$.

2. In the self-consistent development of polar cap arcs, an initial single-arc precipitation pattern splits into multiple arcs. This result suggests that the observed multiple polar cap arcs might not be due to any multiple structures in the magnetospheric source region, but instead, such a multiple feature is internally originated in the dynamical process of the polar cap arcs determined by the whole coupled M-I system.

3. Strong downward currents can develop near the most intense upward currents and form pair structures of field-aligned currents in polar cap arcs. In this specific case, the spatial scale of the pair structures is around tens of kilometers with the downward currents on the morningside.

4. The regions of strong ionization coincide with the regions of intense upward field-aligned currents, but the regions of strong Joule heating appear to have a dawnward shift to the region of the intense upward currents.

5. Our model predicts the existence of plasma flow crossing the polar cap arcs, but the amplitude of such a flow is small, and the characteristic time scale of it is much larger than the time constant for the formation of polar cap arcs.

6. The field-aligned currents of polar cap arcs are mainly closed by Pedersen currents, and the contribution of the Hall component of the field-aligned current is not significant in these polar cap arcs. This is different from the situation of

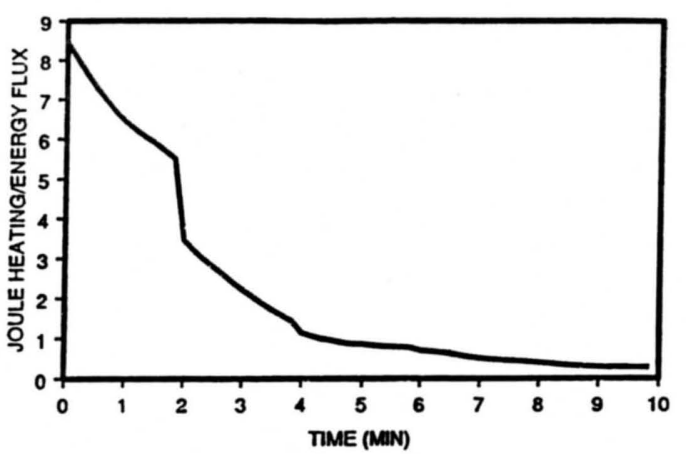

Fig. 14. Temporal evolution of the ratio between the total Joule heating and the total energy flux associated with precipitating electrons in the region which covers the range from $y=-200 \mathrm{~km}$ to $\mathrm{y}=200 \mathrm{~km}$ in the dawn-dusk direction and from $\mathrm{x}=0 \mathrm{~km}$ to 3000 $\mathrm{km}$ in the noon-midnight direction.

auroral arcs in the oval region, where a significant amount of field-aligned currents is closed by Hall currents.

7. Our results show that in the situation of multiple polar cap arcs, the breakup of a single arc is seen first, and a few minutes later, the breakup of a second arc occurs at a distance of tens of kilometers from the first one. Individual arcs move at an average velocity of around $200 \mathrm{~m} / \mathrm{s}$ while their intensities increase.

8. While polar cap arcs are developing, the associated current system tends to become further localized, and at steady state the current system in the narrow regions around the arcs is locally self-closed.

9. Because of the dramatically decreased electric field and greatly enhanced precipitation, the ratio between the total Joule heating and the total energy flux associated with precipitating electrons can be less than 1 in the narrow regions around polar cap arcs.

As a follow-up of this work, a more systematic study of polar cap arcs will be conducted by using the developed model. The future work will focus on the studies of the polar cap arcs with various morphologies, the effects of the solar cycle and seasonal variation, and the influence of various large-scale ionospheric convection patterns on polar cap arcs. The effect of different magnetospheric boundary conditions will be studied, as will the extent of decoupling in the M-I system when allowance is made for $\phi_{\|}$effects.

Acknowledgments. This research was supported by NASA grant NAG5-1484, grant AFOSR-90-0026, and NSF grant ATM-89-13230 to Utah State University.

The Editor thanks W. J. Burke and another referee for their assistance in evaluating this paper.

\section{REFERENCES}

Akasofu, S.-I. The development of the auroral substorm, Planet. Space Sci., 12, 273, 1964.

Akasofu, S.-I., and M. Roederer, Polar cap arcs and the open regions, Planet. Space Sci., 31, 193, 1983.

Alfvén, H., and C.G. Falthammar, Cosmical electrodynamics, Clarendon Press, Oxford, 1963.

Berkey, T., L.L. Cogger, S. Ismail, and Y. Kamide, Evidence for a correlation between Sun-aligned arcs and the interplanetary magnetic field direction, Geophys. Res. Lett., 3, 145, 1976.

Burch, J.L., et al., IMF $B_{y}$-dependent plasma flow and Birkeland currents in the dayside magnetosphere, 1, Dynamics Explorer observations, J. Geophys. Res., 90, 1577, 1985.

Burke, W.J., M.C. Kelley, R.C. Sagalyn, M. Smiddy, and S.T. Lai, Polar cap electric field structures with a northward interplanetary 
magnetic field, Geophys. Res. Lett., 6, 21, 1979.

Burke, W.J., M.S. Gussenhoven, M.C. Kelley, D.A. Hardy, and F.J. Rich, Electric and magnetic field characteristics of discrete arcs in the polar cap, J. Geophys. Res., 87, 2431, 1982.

Cao, F., and J.R. Kan, Reflection of Alfvén waves at an open magnetopause, J. Geophys. Res., 95, 4257, 1990.

Carlson, H.C., Jr., V.B. Wickwar, E.J. Weber, J. Buchau, J.G. Moore, and W. Whiting, Plasma characteristics of polar cap F-layer arcs, Geophys. Res. Lett., 11, 895, 1984.

Carlson, H., A. Heelis, E.J. Weber, and J.R. Sharber, Mesoscale convection patterns during northward interplanetary magnetic field, J. Geophys. Res., 93, 14,501, 1988.

Chiu, Y.T., Formation of polar cap arcs, Geophys. Res. Lett., 16, 743, 1989.

Chiu, T.R., N.U. Crooker, and D.J. Gorney, Model of oval and polar cap arc configurations, J. Geophys. Res., 90, 5153, 1985.

Davis, T.N., The morphology of the polar aurora, J. Geophys. Res., $65,3497,1960$.

Davis, T.N., Negative correlation between polar cap visual aurora and magnetic activity, J. Geophys. Res., 68, 4447, 1963.

Denholm, J.V., and F.R. Bond, Orientation of polar auroras, Aust. J. Phys., 14, 193, 1961.

Frank, L.A., J.D. Craven, J.L. Burch, and J.D. Winningham, Polar views of the Earth's aurora with Dynamic Explorer, Geophys. Res. Lett., 9, 1001, 1982.

Frank, L.A., et al., The theta aurora, J. Geophys. Res., 91, 3177, 1986.

Fridman, M., and J. Lemaire, Relationship between auroral electron fluxes and field-aligned electric potential difference, J. Geophys. Res., 85, 664, 1980.

Goertz, C.K., Kinetic Alfvén waves on auroral field lines, Planet. Space Sci., 32, 1387, 1984.

Gussenhoven, M.S., Extremely high latitude auroras, J. Geophys. Res., 87, 2401, 1982.

Hardy, D.A., W.J. Burke, and M.S. Gussenhoven, DMSP optical and electron measurements in the vicinity of polar cap arcs, J. Geophys. Res., 87, 2413, 1982.

Hoffman, R.A., R.A. Heelis, J.S. Prasad, A Sun-aligned arc observed by DMSP and AE-C, J. Geophys. Res., 90, 9697, 1985.

Ismail, S., and C.-I. Meng, A classification of polar cap auroral arcs, Planet. Space Sci., 30, 319, 1982.

Ismail, S., D.D. Wallis, and L.L. Cogger, Characteristics of polar cap Sun-aligned arcs, J. Geophys. Res., 82, 4741, 1977.

Kan, J.R., and W.J. Burke, A theoretical model of polar cap auroral arcs, J. Geophys. Res., 90, 4171, 1985.

Kan, J.R., and W. Sun, Simulation of the westward traveling surge and Pi2 pulsations during substorms, J. Geophys. Res., 90, 10,911, 1985.

Kan, J.R., L. Zhu, and S.-I. Akasofu, A theory of substorms: Onset and subsidence, J. Geophys. Res., 93, 5624, 1988.

Lassen, K., and C. Danielsen, Quiet time pattern of auroral arcs for different directions of the interplanetary magnetic field in the $Y-Z$ plane, J. Geophys. Res., 83, 5277, 1978.

Lyons, L.R., Generations of large-scale regions of auroral currents, electric potentials, and precipitation by the divergence of the convection electric field, J. Geophys. Res., 85, 17, 1980.

Lysak, R.L., Coupling of the dynamic ionosphere to auroral flux tubes, J. Geophys. Res., 91, 7047, 1986.

Lysak, R.L., Feedback instability of the ionospheric resonant cavity, J. Geophys. Res., 96, 1553, 1991.

Lysak, R.L., and C.T. Dum, Dynamics of magnetosphere-ionosphere coupling including turbulent transport, J. Geophys. Res., 88, 365, 1983.

Maezawa, K., Magnetospheric convection induced by the positive and negative $Z$ components of the interplanetary magnetic field: Quantitative analysis using polar cap magnetic records, J. Geophys. Res., 81, 2289, 1976.

Makita, K., C.-I. Meng, and S.-I. Akasofu, Transpolar auroras, their particle precipitation, and IMF $B_{y}$ component, J. Geophys. Res., 96, 14,085, 1991.
Mallinckrodt, A.J., and C.W. Carlson, Relations between transverse electric fields and field-aligned currents, J. Geophys. Res., 83, 1426, 1978.

Maltsev, Y.P., W.B. Lyatsky, and A.M. Lyatskaya, Currents over the auroral arc, Planet. Space Sci., 25, 53, 1977.

Marklund, G.T., and L.G. Blomberg, On the influence of localized electric fields and field-aligned currents associated with polar arcs on the global potential distribution, J. Geophys. Res., 96, 13,977, 1991.

Mende, S.B., J.H. Doolittle, R.M. Robinson, R.R. Vondrak, and F.J. Rich, Plasma drifts associated with a system of Sun-aligned arcs in the polar cap, J. Geophys. Res., 93, 256, 1988.

Meng, C.-I., Polar cap arcs and the plasma sheet, Geophys. Res. Lett. 8, 273, 1981.

Murphree, J.S., C.D. Anger, and L.L. Cogger, The instantaneous relationship between polar cap and oval auroras at times of northward interplanetary magnetic field, Can. J. Phys., 60, 349, 1982.

Nielsen, E., J.D. Craven, L.A. Frank, and R.A. Heelis, Ionospheric flows associated with a transpolar arc, J. Geophys. Res., 95, 21,169, 1990.

Obara, T., M. Kitayama, T. Mukai, N. Kaya, J.S. Murphree, and L.L. Cogger, Simultaneous observations of Sun-aligned polar cap arcs in both hemispheres by EXOS-C and Viking, Geophys. Res. Lett., 15, $713,1988$.

Peterson, W.K., and E.G. Shelley, Origin of the plasma in a cross-polar cap auroral feature (theta aurora), J. Geophys. Res., 89, 6729, 1984.

Potemra, T.A., L.J. Zanetti, P.F. Bythrow, A.T.Y. Lui, and T. Iijima, $B$-dependent convection patterns during northward interplanetary magnetic field, J. Geophys. Res., 89, 9753, 1984.

Rasmussen, C.E., and R.W. Schunk, Ionospheric convection driven by NBZ currents, J. Geophys. Res., 92, 4491, 1987.

Rees, M.H., Auroral ionization and excitation by incident energetic electrons, Planet. Space Sci., 11, 1209, 1963.

Reiff, P.H., and J.L. Burch, IMF $B_{y}$-dependent dayside plasma flow and Birkeland currents in the dayside magnetosphere, 2, A global model for northward and southward IMF, J. Geophys. Res., 90, 1595, 1985.

Rich, F.J., D.A. Hardy, R.H. Redus, and M.S. Gussenhoven, Northward IMF and pattems of high-latitude precipitation and field-aligned currents: The February 1986 storm, J. Geophys. Res., 95, 7893, 1990.

Rothwell, P.L., M.B. Silevitch, and L.P. Block, A model for the propagation of the westward traveling surge, J. Geophys. Res., 89, $8941,1984$.

Russell, C.T., The configuration of the magnetosphere, in Critical Problems of Magnetospheric Physics, vol. 1, edited by E.R. Dyer, p. 1149, National Academy of Sciences, Washington, D.C., 1972.

Valladares, C.E., and H.C. Carlson, Jr., The electrodynamic, thermal, and energetic character of intense Sun-aligned arcs in the polar cap, $J$. Geophys. Res., 96, 1379, 1991.

Walls, F.L., and G.H. Dunn, Measurement of total cross-section for electron recombination with $\mathrm{NO}^{+}$and $\mathrm{O}_{2}^{-}$using ion storage technology, J. Geophys. Res., 79, 1911, 1974.

Weber, E.J., and J. Buchau, Polar cap F-layer auroras, Geophys. Res. Lett., 8, 125, 1981.

Weill, G., Aspects de l'aurore observée à la base Dumont-d'Urville en Terre Adelie, CR. Acad. Sci., 246, 2925, 1958.

Zhu, L., and J.R. Kan, Effects of ionospheric recombination time scale on the auroral signature of substorms, J. Geophys. Res., 95, 10,389, 1990.

D. J. Crain, R. W. Schunk, J. J. Sojka, and L. Zhu, Center for Atmospheric and Space Sciences, Utah State University, Logan, UT 84322-4405.
(Received December 27, 1991; revised May 1, 1992; accepted June 10, 1992.) 